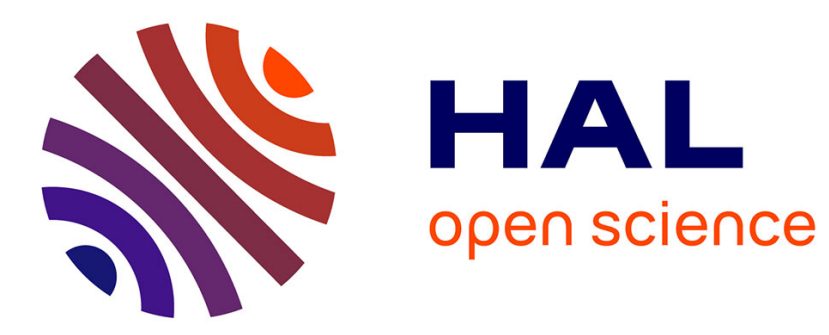

\title{
Étude expérimentale de la diffusion Rayleigh-Brillouin dans le méthane
}

\author{
A.-M. Cazabat, P. Lallemand
}

\section{To cite this version:}

A.-M. Cazabat, P. Lallemand. Étude expérimentale de la diffusion Rayleigh-Brillouin dans le méthane. Journal de Physique Lettres, 1975, 36 (2), pp.29-33. 10.1051/jphyslet:0197500360202900 . jpa00231147

\section{HAL Id: jpa-00231147 https://hal.science/jpa-00231147}

Submitted on 1 Jan 1975

HAL is a multi-disciplinary open access archive for the deposit and dissemination of scientific research documents, whether they are published or not. The documents may come from teaching and research institutions in France or abroad, or from public or private research centers.
L'archive ouverte pluridisciplinaire HAL, est destinée au dépôt et à la diffusion de documents scientifiques de niveau recherche, publiés ou non, émanant des établissements d'enseignement et de recherche français ou étrangers, des laboratoires publics ou privés. 


\title{
ÉTUDE EXPÉRIMENTALE DE LA DIFFUSION RAYLEIGH-BRILLOUIN DANS LE MÉTHANE
}

\author{
A.-M. CAZABAT et P. LALLEMAND \\ Laboratoire de Spectroscopie Hertzienne \\ Ecole Normale Supérieure, 24, rue Lhomond, 75231 Paris Cedex 05, France
}

(Reşu le 3 octobre 1974, accepté le 12 décembre 1974)

\begin{abstract}
Résumé. - Les spectres de diffusion de la lumière par le méthane obtenus dans un assez large domaine de pression sont comparés à plusieurs modèles théoriques dus à Desai et Coll. en vue de déterminer une valeur approchée du nombre moyen $Z$ de collisions nécessaires pour modifier l'état rotationnel des molécules. On utilise pour des pressions assez faibles les solutions approchées de l'équation de Wang-Chang et Uhlenbeck obtenues par la méthode des modèles, et l'hydrodynamique translationnelle à plus haute pression où il faut tenir compte du fait qu'on n'a pas un gaz parfait. On trouve un bon accord entre les spectres expérimentaux et les spectres théoriques à condition de prendre $Z=9,7 \pm 0,7$ qui est proche de la valeur déduite de la viscosité de volume obtenue à partir de mesures de l'absorption ultrasonore.
\end{abstract}

\begin{abstract}
Light scattering spectra have been obtained for methane in a fairly wide pressure range and have been compared with several theoretical models due to Desai et al., in order to determine the value of the mean number $Z$ of collisions necessary to change the rotational state of the molecules. At low pressure, the Wang-Chang and Uhlenbeck equation solved by the model method has been used, whereas at higher pressure where departures from a perfect gas are not negligible, the translational hydrodynamics is used. Good agreement has been found between experimental and theoretical spectra provided one takes $Z=9.7 \pm 0.7$ which is close to the value derived from bulk viscosity obtained through ultrasonic attenuation measurements.
\end{abstract}

Introduction. - Les dernières années ont vu le développement rapide des études sur la diffusion de la lumière dans les gaz. Si actuellement le gaz monoatomique est bien connu, tant théoriquement [1] qu'expérimentalement [2], dans le domaine cinétique aussi bien que dans le domaine hydrodynamique, il n'en est pas de même des gaz polyatomiques. Les théories actuelles visent à décrire correctement les transferts d'énergie entre la translation et les mouvements internes des molécules. Ce problème se complique particulièrement lorsque les échelles de temps pour ces transferts d'énergie se situent dans le domaine intermédiaire entre les comportements hydrodynamique et cinétique du gaz.

En effet, le domaine optimum de fréquence pour l'étude d'un phénomène de temps caractéristique $\tau$ est celui où $\omega \tau \sim 1$. ( $\omega$ est ici la pulsation $2 \pi v, v$ la fréquence.) Dans le cas des transferts d'énergie rotationtranslation, le temps caractéristique $\tau_{\text {rot }}$, qui est le temps de relaxation de l'énergie de rotation par couplage avec la translation, est du même ordre de grandeur que le temps moyen entre collisions cinétiques dans le gaz. Soit $\tau_{\mathrm{c}}$ ce dernier; on le calcule approximativement par la formule de théorie cinétique des gaz [3]

$$
\tau_{\mathrm{c}} \sim 1,27 \frac{\eta_{\mathrm{s}}}{P}
$$

où $\eta_{\mathrm{s}}$ est la viscosité de cisaillement et $P$ la pression. Cette dernière est le paramètre qui variera d'une expérience à l'autre, car nous prenons des séries de spectres à angle de diffusion $\theta$ constant, donc en négligeant les variations d'indice de réfraction du gaz, à vecteur d'onde de diffusion $k$ constant.

En conséquence, l'étude de la relaxation de rotation aura lieu dans le domaine où $\omega \tau_{\mathrm{c}} \sim 1$ qui marque le passage du régime hydrodynamique (haute pression) au régime cinétique (basse pression). On peut aussi dire, en utilisant une formulation équivalente, que le paramètre $Y$ défini par

$$
\mathrm{Y}=\frac{n k_{\mathrm{B}} T}{\sqrt{2} v_{0} k \eta_{\mathrm{s}}}
$$

est alors de l'ordre de 1. Dans cette formule, $k_{\mathrm{B}}$ est la constante de Boltzmann, $n$ la densité (en nombre 
de molécules par unité de volume), $v_{0}$ la vitesse moléculaire donnée par $v_{0}=\left(k_{\mathrm{B}} T / m\right)^{1 / 2}$ où $m$ est la masse moléculaire et $T$ la température absolue. La valeur $Y=1$ est obtenue pour une pression de 0,27 bar dans nos conditions expérimentales. Le but de notre étude est la détermination du rapport $\tau_{\text {rot }} / \tau_{\mathrm{c}}$ que nous appellerons $Z$, et qui représente le nombre moyen de collisions nécessaires pour modifier l'état interne de rotation des molécules. Outre la diffusion de la lumière, il existe d'autres méthodes expérimentales permettant d'évaluer $Z$. La plus précise est la mesure de l'atténuation ultrasonore supplémentaire due au phénomène de relaxation, et qu'on décrit au moyen d'une viscosité de volume. C'est aux valeurs de $Z$ obtenues par cette méthode que nous comparerons nos résultats.

Nous utiliserons lors du dépouillement des résultats expérimentaux deux types de théories : d'une part les modèles cinétiques de Boley, Desai et Tenti [4] et d'autre part l'hydrodynamique translationnelle de Weinberg, Kapral et Desai [5]. Renvoyant le lecteur aux articles originaux de ces auteurs, nous ne donnerons ici qu'un très bref aperçu sur ces théories.

Boley, Desai et Tenti [4] appliquent l'équation de Wang-Chang et Uhlenbeck [6] au cas simplifié d'un gaz présentant un seul temps de relaxation pour les degrés de liberté internes. Ils calculent de manière approchée la fonction d'autocorrélation $\left\langle\rho_{k}(t) \rho_{-k}(0)\right\rangle$ de la composante de Fourier de vecteur $k$ de la densité du gaz. Cette résolution se fait par la méthode des modèles qui consiste à remplacer l'opérateur de collision linéarisé par une expression approchée. Nous utiliserons ici deux approximations : la première est un modèle à 7 moments que nous noterons $S_{7}$, la seconde n'en comporte que 6 mais lui est supérieure pour des raisons de symétrie (il donne en tout cas de meilleurs résultats quand on l'applique à un gaz monoatomique [7], ce qui permet de dire que la partie translationnelle des mouvements moléculaires sera bien décrite). Il sera noté $S_{6}$. Ces modèles couvrent le domaine cinétique et en principe le domaine hydrodynamique, mais ils sont limités ici au début du régime hydrodynamique car ils ne comportent pas de correction de non-idéalité du gaz.

Lorsque la pression augmente, il est nécessaire de tenir compte de ces corrections. C'est pourquoi nous relayons alors les théories $S_{6}$ et $S_{7}$ par l'hydrodynamique translationnelle de Weinberg, Kapral et Desai [5] que nous noterons WKD. Cette théorie provient de la description du fluide au moyen de l'équation de Langevin généralisée en utilisant comme ensemble de variables une combinaison linéaire des trois variables usuelles (densité, température et divergence de la vitesse) et d'une variable représentant l'énergie interne des molécules. La différence essentielle de cette théorie par rapport à celle de Mountain [8] est que l'énergie interne peut relaxer non seulement par collisions avec un temps caractéristique $\tau$ mais aussi par diffusion des molécules excitées. Il en résulte que la raie de Mountain caractéristique des phénomènes de relaxation n'a plus une largeur de l'ordre de $\tau^{-1}$, mais une largeur de l'ordre de $\tau^{-1}+D k^{2}$ où $D$ est le coefficient de self-diffusion des molécules excitées. Cette théorie ne couvre pas le domaine cinétique et est médiocre dans le domaine intermédiaire, mais il est aisé d'y introduire des corrections de non-idéalité. Ces différents types de théories se raccordent sans problème et assurent en principe une description correcte du gaz à toutes les pressions utilisées.

Montage et spectres expérimentaux. - La source lumineuse est un laser à argon ionisé, rendu monomode à la longueur d'onde de $5145 \AA$ et d'intensité voisine de $300 \mathrm{~mW}$. La diffusion est étudiée à un angle voisin de $30^{\circ}$, l'analyse spectrale étant réalisée au moyen d'un interféromètre Fabry-Pérot sphérique confocal balayé piézoélectriquement. L'interordre est $1500 \mathrm{MHz}$, et la finesse globale du montage 50 ou plus. Le signal est recueilli sur un photomultiplicateur EMI 9635 refroidi, traité par une chaîne de comptage et reçu sur un enregistreur. Le montage ne comporte pas de dispositif d'accumulation.

Le méthane a été étudié entre 0,75 et 25 bar. Nous avons vérifié dans tous les cas l'absence de lumière parasite en effectuant après chaque spectre un enregistrement cellule vidée. Aucun fond continu dépolarisé n'a été décelé. Enfin, le fond continu parasite a été mesuré en coupant le faisceau laser et soustrait aux courbes expérimentales.

Pour les pressions supérieures à l'atmosphère, le dépouillement a été conduit de la façon suivante : on a relevé pour chaque interordre les rapports $R / B$ et $R B / B$ (définis sur la figure 1) ainsi que le déplacement Brillouin $\Delta B$. (Nous avons défini ce dernier par la position du sommet de la raie Brillouin, ce qui est une convention inhabituelle.) Cette méthode permet de vérifier l'absence de valeurs aberrantes et de tracer, en fonction de la pression, les courbes représentant les trois paramètres $R / B, R B / B$ et $\Delta B$. Par sa grande sécurité, cette étude globale est plus avantageuse que l'examen d'un spectre unique. Nous avons ensuite effectué la convolution des spectres théoriques et de la fonction d'appareil expérimentale, puis tracé les réseaux théoriques

$$
\frac{R}{B}(P), \frac{R B}{B}(P) \quad \text { et } \quad \Delta B(P)
$$

avec $P$ comme variable et $Z$ comme paramètre. Ces réseaux théoriques sont alors comparés aux courbes expérimentales.

Aux pressions inférieures à l'atmosphère, le relevé à la main devient imprécis car les raies sont très larges. Nous n'avons guère étudié ce domaine qui est peu sensible à la valeur de $Z$ car $\omega \tau_{\text {rot }}$ est supérieur à 3 . Nous nous sommes contentés de comparer aux spectres théoriques (avec convolution) nos quelques spectres expérimentaux et avons constaté un bon accord avec les théories $S_{6}$ et $S_{7}$ qui diffèrent peu dans ce domaine. 


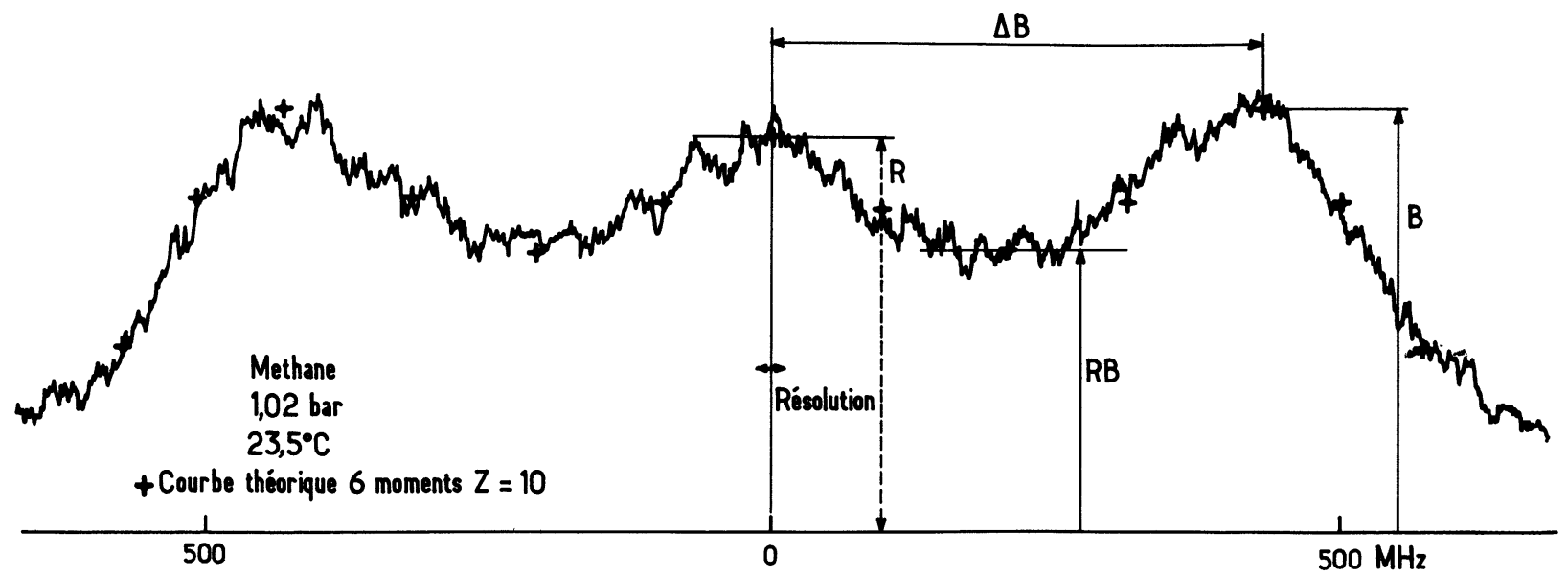

Fig. 1. - Spectre de la lumière diffusée par $\mathrm{CH}_{4}$ pour un angle de diffusion de $29,3^{\circ}$, à $23,5^{\circ} \mathrm{C}$ et 1,02 bar. Les croix ont été calculées à partir du modèle théorique $\mathrm{S}_{6}$ pour $Z=10$. Ce spectre comporte les définitions des quantités $R, B, R B$ et $B$ utilisées par ailleurs.

Analyse des résultats obtenus. - Nous avons utilisé les théories $S_{6}$ et $S_{7}$ jusqu'à 3 bar. Au-delà, la théorie WKD est nécessaire. Nous y avons effectué les corrections de non-idéalité du gaz : coefficients de transport et chaleurs spécifiques dépendant de la pression, ainsi que la quantité $P V / R T$ ( $V$ volume du gaz, $R$ constante des gaz parfaits). Ces diverses corrections ont été déduites de valeurs publiées [9].

L'interprétation des courbes expérimentales exige quelques précautions préalables. Si le pointé des maxima Brillouin est précis à mieux de $1 \%$ près, il n'en est pas de même du déplacement théorique correspondant car l'angle de diffusion n'est pas connu avec une précision suffisante. Nous avons constaté sur ce dernier une incertitude de $\pm 0,7^{\circ}$ (pour $\theta \simeq 30^{\circ}$ ), ce qui conduit à une incertitude de $\pm 3 \%$ sur la valeur théorique de $\Delta B$. Cette quantité ne pourra donc pas être utilisée lors de la comparaison théorieexpérience. L'examen des réseaux théoriques montre que $\Delta B$ dépend beaucoup de l'angle de diffusion, mais est peu sensible à $Z$. Le rapport $R / B$ au contraire dépend peu de l'angle $\theta$ mais est très sensible à $Z$. (En ce qui concerne $R B / B$, il est fort peu sensible à l'un et à l'autre : nous avons vérifié l'accord entre l'unique courbe théorique et notre courbe expérimentale, mais nous n'en avons pas tiré de renseignement sur $Z$.) La différence de comportement des quantités $\Delta B$ et $R / B$ permet donc de lever toutes les incertitudes : du réseau $R / B$ on tire une valeur approchée de $Z$. Celle-ci suffit à déterminer $\theta$ à l'aide du réseau $\Delta B$. Le dépouillement est alors réalisé à l'aide de cette valeur de $\theta$ et on constate que la valeur de $Z$ donnée par $R / B$ n'est pas sensiblement modifiée. Ce traitement a été nécessaire pour une série de courbes, obtenues à $29,3^{\circ}$, pour lesquelles la pression était inférieure à 5 bar. Les points obtenus audessus de 5 bar n'ont pas nécessité de correction, l'angle de diffusion étant suffisamment proche de $30^{\circ}$.

Les trois théories utilisées ont été établies dans le cas de la relaxation de l'énergie interne dans un système à deux niveaux. Ce n'est pas le cas du méthane, aussi la valeur de $Z$ que nous obtenons doitelle être considérée comme une moyenne sur les différents niveaux de rotation. En particulier, elle doit être proche de la valeur 8,7 obtenue récemment [10] par les mesures d'absorption ultrasonore, mais il n'est pas évident qu'elle lui soit égale.

Par ailleurs, ces théories supposent que les coefficients de transport du gaz vérifient la formule d'Eucken modifiée [11]

$$
f=\frac{\lambda M}{\eta_{\mathrm{s}} C_{\mathrm{v}}}=\frac{15}{4} \frac{R}{C_{\mathrm{v}}}+\frac{\rho D}{\eta_{\mathrm{s}}} \frac{C_{\mathrm{Int}}}{C_{\mathrm{v}}}
$$

$\lambda$ conductivité thermique, $M$ masse molaire, $\rho$ densité et $D$ coefficient de self-diffusion, ou encore que

$$
\lambda=\lambda_{\mathrm{tr}}+\rho C_{\mathrm{Int}} D
$$

$\lambda_{\mathrm{tr}}$ conductivité thermique du gaz monoatomique

$$
\lambda_{\mathrm{tr}}=\frac{15}{4} R \frac{\eta_{\mathrm{s}}}{M}
$$

La formule d'Eucken n'est valable que lorsque $Z$ est assez grand devant 1 , sinon il faut utiliser la formule plus complète de Mason et Monchick [12]. Dans le cas du méthane, on vérifie que le terme correctif est négligeable. L'utilisation de ces théories est donc justifiée.

Le dernier point à souligner est l'existence à température ordinaire d'une contribution vibrationnelle non négligeable à la chaleur spécifique : $C_{\mathrm{vib}}=0,5 \mathrm{cal} /$ mole $K$. Cette contribution doit être retranchée dans les calculs car aux fréquences étudiées la vibration est bloquée. $C_{\mathrm{p}}$ et $C_{\mathrm{v}}$ sont donc corrigés, mais aussi $\lambda$

$$
\lambda_{\text {réel }}=\lambda_{\text {mesuré à } \omega=0}-\rho C_{\text {vib }} D .
$$

La correction est de 6,7\%. La valeur de $Z$ est modifiée de $10 \%$ par rapport à ce qu'elle serait sans correction sur $\lambda$, mais en corrigeant $C_{\mathrm{p}}$ et $C_{\mathrm{v}}$. 
Les figures 2, 3 et 4 illustrent la comparaison entre courbes théoriques et courbes expérimentales. On constate que l'accord est bon et que les deux types de théories conduisent bien aux mêmes valeurs de $Z$.

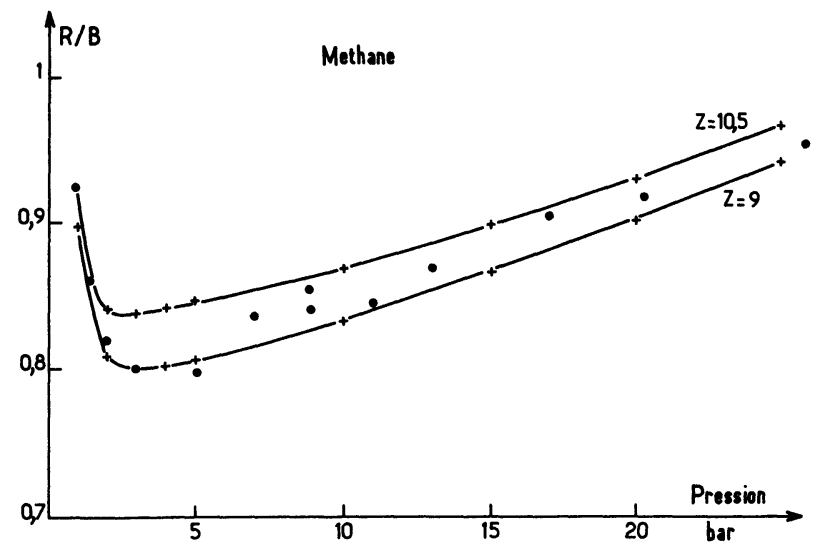

Fig. 2. - Valeurs expérimentales ( $\bullet$ ) et théoriques $(+)$ du rapport de l'intensité maximum de la raie Rayleigh et de l'intensité maximum de la raie Brillouin dans le méthane. Les points théoriques ont été calculés à partir de l'hydrodynamique translationnelle.

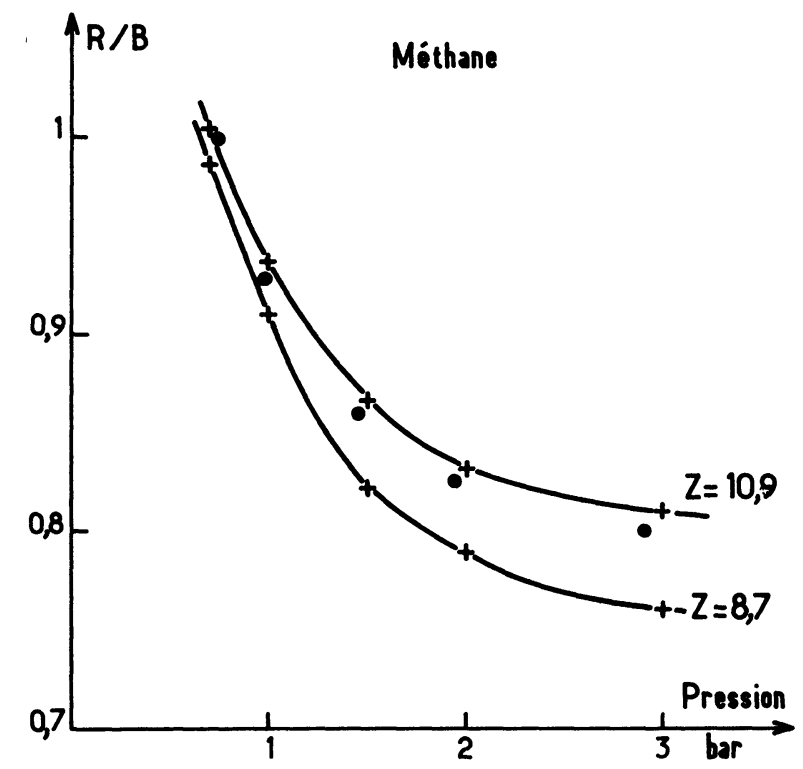

Fig. 3. - Valeurs expérimentales $(\bullet)$ et théoriques $(+)$ du rapport de l'intensité maximum de la raie Rayleigh et de l'intensité maximum de la raie Brillouin dans le méthane. Les points théoriques ont été calculés à partir de la théorie $S_{6}$.

Nous pouvons estimer $9 \leqslant Z \leqslant 10,5$. Il serait illusoire d'exiger des courbes plus de précision car elles sont limitées vers les basses pressions par la détérioration du rapport signal/bruit, et vers les hautes pressions par l'importance croissante des corrections de non-idéalité du gaz. On peut considérer cette valeur de $Z$ comme satisfaisante en référence

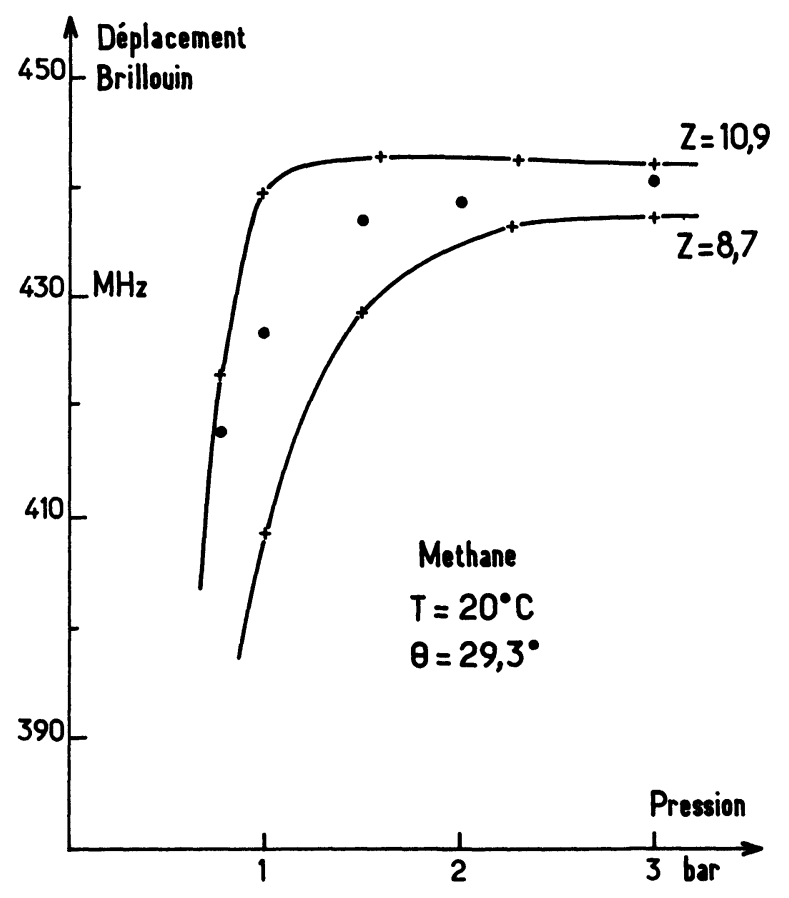

Fig. 4. - Valeurs expérimentales (๑) et théoriques $(+)$ du déplacement Brillouin dans le méthane à $20^{\circ} \mathrm{C}$ pour un angle de diffusion de $29,3^{\circ}$. Les valeurs théoriques ont été calculées à partir de la théorie $S_{6}$.

aux données d'absorption ultrasonore bien que, nous l'avons déjà dit, les quantités mesurées ne soient pas nécessairement tout à fait identifiables.

Il serait souhaitable d'améliorer le montage afin de pouvoir mesurer précisément les largeurs de raie et notamment d'en déduire la valeur de $\lambda_{\text {réel }}$ sans passer par la formule (1) dans laquelle le coefficient $D$ est très discuté. Il ne semble en effet pas évident que le coefficient de diffusion soit le même dans un état excité que dans l'état fondamental où on le mesure habituellement. Ce problème sans importance dans $\mathrm{CH}_{4}$ devient gênant dans $\mathrm{CO}_{2}$ et surtout $\mathrm{SF}_{6}$ [13] pour lesquels nous avons des résultats qui seront publiés par ailleurs.

Les résultats présentés ici, ainsi que ceux de Hubert et May [14] montrent que l'on dispose maintenant d'une bonne description des fluctuations de densité dans les gaz polyatomiques $\mathrm{H}_{2}$ et $\mathrm{CH}_{4}$, mais il faudra faire des mesures dans d'autres gaz avant de pouvoir donner des conclusions définitives sur la valeur des modèles utilisés ici.

Les calculs utilisant les théories $S_{6}$ et $S_{7}$ ont été exécutés à partir d'un programme prêté par $\mathrm{R}$. C. Desai et adapté à notre ordinateur. Nous sommes heureux de lui renouveler ici l'expression de notre gratitude.

\section{Bibliographie}

[1] YIP, S., J. Acoust. Soc. Amer. 49 (1971) 941 et références données dans cet article de revue.

[2] Clark, N. A., Ph. D. Thesis MIT 1970, non publié.
[3] Chapman, S. et Cowling, T. G., The Mathematical Theory of Non-Uniform Gases, $3^{\mathrm{e}}$ édition (Cambridge University Press) 1970, p. 226. 
[4] Boley, C. D., Desai, R. C. et Tenti, G., Can. J. Phys. 50 (1972) 2158.

[5] Weinberg, M., Kapral, R. et Desai, R. C., Phys. Rev. A 7 (1973) 1413.

[6] Voir une introduction à la théorie cinétique des gaz polyatomiques dans Studies in Statistical Mechanics édité par J. de Boer et G. E. Uhlenbeck (North Holland, Amsterdam) 1964, Vol. 2.

[7] Tenti, G., Boley, C. D. et Desai, R. C., Can. J. Phys. 52 (1974) 285.

[8] Mountain, R. D., J. Res. Nat. Bur. Stand. 72A (1968) 95.

[9] Michels, A. et Nederhagt, G. W., Physica 3 (1936) 569.

[10] Prangsma, G. J., Alberga, A. H. et Beenakker, J. J. M., Physica 64 (1973) 278. La relaxation de rotation introduit dans l'expression de l'amortissement ultrasonore un terme supplémentaire de viscosité de volume $\eta_{v}$ avec

$$
\tau_{\mathrm{rot}}=\frac{C_{v}^{2}}{R C_{\mathrm{rot}}} \frac{\eta_{v}}{P}
$$

$C_{v}$ chaleur spécifique à volume constant, $C_{\text {rot }}$ chaleur spécifique de rotation, donc

$$
Z=1,27 \frac{C_{v}^{2}}{R C_{\text {rot }}} \frac{\eta_{v}}{\eta_{\mathrm{s}}}
$$

[11] HiRsChfelder, J., CURTISs, C. et BIRD̆, R., Molecular Theory of gases and liquids (J. Wiley, N. Y.) 1954, p. 501.

[12] Mason, E. A. et Monchick, L., J. Chem. Phys. 36 (1962) 1622.

[13] Clark, N. A., Mellman, G. R. et Greytak, T. J., Phys. Rev. Lett. 29 (1972) 150.

[14] Hubert, M. et MaY, A. D., Can. J. Phys. (1974) à paraître. 